\title{
SPMLMI: Predicting IncRNA-miRNA interactions in humans using a structural perturbation method
}

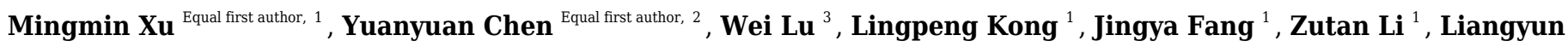 \\ Zhang ${ }^{\text {Corresp., } 2 \text {, Cong Pian }}{ }^{\text {Corresp. } 2}$ \\ ${ }^{1}$ College of Agriculture, Nanjing Agricultural University, Nanjing, Jiangsu, China \\ 2 College of Sciences, Nanjing Agricultural University, Nanjing, Jiangsu, China \\ 3 College of Life Sciences, Nanjing Agricultural University, Nanjing, Jiangsu, China \\ Corresponding Authors: Liangyun Zhang, Cong Pian \\ Email address: zlyun@njau.edu.cn, piancong@njau.edu.cn
}

Long non-coding RNA (IncRNA)-microRNA (miRNA) interactions are quickly emerging as important mechanisms underlying the functions of non-coding RNAs. Accordingly, predicting IncRNA-miRNA interactions provides an important basis for understanding the mechanisms of action of ncRNAs. However, the accuracy of the established prediction methods is still limited. In this study, we used structural consistency to measure the predictability of interactive links based on a bilayer network by integrating information for known IncRNA-miRNA interactions, an IncRNA similarity network, and an miRNA similarity network. In particular, by using the structural perturbation method, we proposed a framework called SPMLMI to predict potential IncRNA-miRNA interactions based on the bilayer network. We found that the structural consistency of the bilayer network was higher than that of any single network, supporting the utility of bilayer network construction for the prediction of IncRNA-miRNA interactions. Applying SPMLMI to three real datasets, we obtained areas under the curves of $0.9512 \pm 0.0034,0.8767 \pm 0.0033$, and $0.8653 \pm 0.0021$ based on 5 -fold cross-validation, suggesting good model performance. In addition, the generalizability of SPMLMI was better than that of the previously established methods. Case studies of two IncRNAs (i.e., SNHG14 and MALAT1) further demonstrated the feasibility and effectiveness of the method. Therefore, SPMLMI is a feasible approach to identify novel IncRNA-miRNA interactions underlying complex biological processes. 


\section{SPMLMI: Predicting IncRNA-miRNA interactions in}

\section{2 humans using a structural perturbation method}

3

4

5

6

7

9

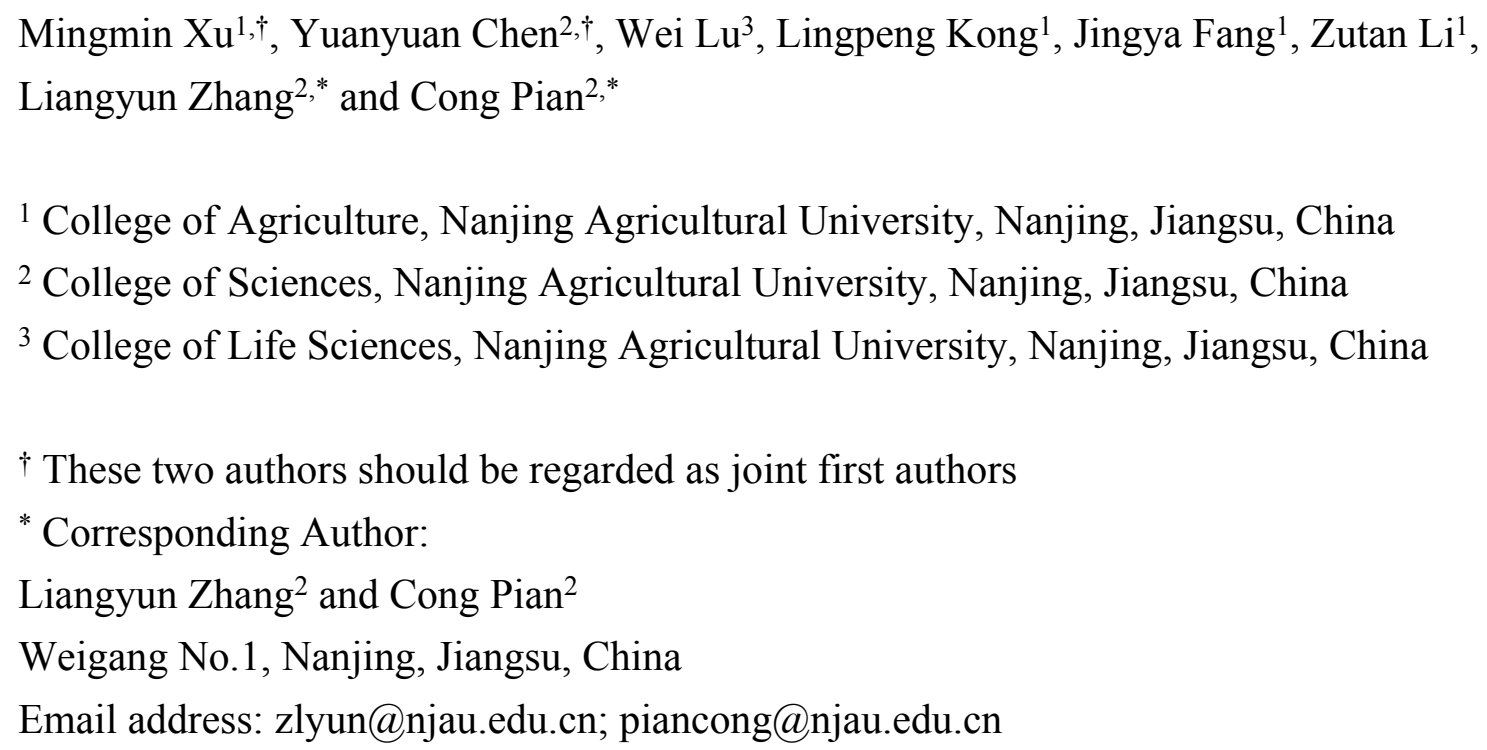

\section{Abstract}

Long non-coding RNA (lncRNA)-microRNA (miRNA) interactions are quickly emerging as important mechanisms underlying the functions of non-coding RNAs. Accordingly, predicting lncRNA-miRNA interactions provides an important basis for understanding the mechanisms of action of ncRNAs. However, the accuracy of the established prediction methods is still limited. In this study, we used structural consistency to measure the predictability of interactive links based on a bilayer network by integrating information for known lncRNA-miRNA interactions, an lncRNA similarity network, and an miRNA similarity network. In particular, by using the structural perturbation method, we proposed a framework called SPMLMI to predict potential lncRNA-miRNA interactions based on the bilayer network. We found that the structural consistency of the bilayer network was higher than that of any single network, supporting the utility of bilayer network construction for the prediction of lncRNA-miRNA interactions. Applying SPMLMI to three real datasets, we obtained areas under the curves of $0.9512 \pm 0.0034$, $0.8767 \pm 0.0033$, and $0.8653 \pm 0.0021$ based on 5-fold cross-validation, suggesting good model performance. In addition, the generalizability of SPMLMI was better than that of the previously established methods. Case studies of two lncRNAs (i.e., SNHG14 and MALAT1) further demonstrated the feasibility and effectiveness of the method. Therefore, SPMLMI is a feasible 
35

36

37

38

39

40

41

42

43

44

45

46

47

48

49

50

51

52

53

54

55

56

57

58

59

60

61

62

63

64

65

66

67

68

69

approach to identify novel lncRNA-miRNA interactions underlying complex biological processes.

\section{Introduction}

For a long time, our framework for understanding the nature of genetic programming in complex organisms was biased by the assumption that protein-coding genes are the majority of the bearers of genetic information. Researchers have recently abandoned their notions about the regulation of complex cellular mechanisms, shifting their focus to previously unexplored RNAs. We now know that RNAs are functionally versatile molecules directly involved in the regulation of many cellular processes, such as epigenetic control, gene transcription, translation, RNA transformation, chromosome organization, and genome defense as well as cell proliferation and developmental programs (Guil \& Esteller 2015; Morris \& Mattick 2014). In most instances, the key regulatory factors are non-coding RNAs (ncRNAs) (Huntzinger \& Izaurralde 2011; Rinn \& Chang 2012; Sabin et al. 2013), which account for the vast majority of mammalian transcripts and range in length from 22 nucleotides to hundreds of kilobases. Although there are established principles that define classes of ncRNAs (e.g., tRNAs and miRNAs), it could be suggested that each ncRNA has a unique function (Cech \& Steitz 2014).

The first miRNA (microRNA) was reported in 1993 as a short RNA in Caenorhabditis elegans able to control the timing of developmental transitions by base pairing to partially complementary sequences in the 3'-UTR of its target mRNA (Lee et al. 1993; Wightman et al. 1993). Today, miRNAs are well-known gene expression repressors (Béthune et al. 2012; Braun et al. 2013; Huntzinger \& Izaurralde 2011), and are widely found in human and plant genomes (Krol et al. 2010; Voinnet 2009). LncRNAs (long non-coding RNAs) are the most abundant ncRNAs; tens of thousands of human lncRNAs have been identified. However, the roles of most lncRNAs in cellular processes have yet to be determined, and we anticipate that many new functions will be discovered (Quinn \& Chang 2016; Chen et al. 2019a; Chen et al. 2017).

Previous studies have concluded that lncRNAs could regulate gene expression patterns by biomolecular interactions, including lncRNA-protein, lncRNA-mRNA, and lncRNA-ncRNA interactions (Li et al. 2014). Yoon et al. (2014) reviewed functional interactions among miRNAs and lncRNAs with important roles in gene expression programs. We believe that detailed analyses of the interactivity between lncRNAs and miRNAs will enrich our understanding of the functions of cellular networks.

RNA-RNA interactions are rapidly emerging as one of the most important functional mechanisms involving ncRNAs (Lai \& Meyer 2016). Typically, the discovery of molecular interactions in biological network requires extensive experimental work (Clauset et al. 2008). 
70 Biological experiments are highly reliable for studies of biomolecular interactions; however, they

71 are difficult to carry out on a large scale owing to the high costs and long execution times. An

72 alternative to studying interactions between each pair of molecules in a huge and complex

73 biological network is a predictive approach, in which prior interaction information is used to

74 identify molecules that are most likely to be related, providing a basis for further experimental

75 studies. In particular, computational prediction methods are useful for prioritizing candidate

76 lncRNA-miRNA interactions on a large scale.

77 Most existing RNA-RNA interaction prediction methods are based on sequence information,

78 including sequence conservation, seed region matching, site accessibility, and minimum free

79 energy, and predict matching relationships between miRNAs, small RNAs, and small nucleolar

80 RNAs with their targets (mRNAs and rRNAs) (Lai \& Meyer 2016; Quinn \& Chang 2016).

81 Different from mRNAs and rRNAs, lncRNAs have substantial sequence variation and relatively

82 low conservation, and they are involved in relatively complex molecular interactions.

83 Accordingly, we cannot predict interactions between lncRNAs and miRNAs by sequence

84 information alone.

As a primary type of competitive endogenous RNA (ceRNA), lncRNAs can act as miRNA sponges to weaken the effect of miRNAs on mRNAs; therefore, miRNAs play important roles in the molecular mechanism of lncRNAs (Salmena et al. 2011; Xu et al. 2015; Thomson \& Dinger 2016; Lv et al. 2020). ceRNA crosstalk appears to be closely related to miRNA expression levels, and the specificity of interactions may depend on the expression profile of miRNAs (Ala et al. 2013; Xu et al. 2015; Xu et al. 2016). In addition, some lncRNAs may be co-regulated in expression networks, suggesting that multiple lncRNAs may interact with specific miRNA clusters to regulate biological processes in a coordinated way (Li et al. 2015; Thomson \& Dinger 2016). It is therefore reasonable to expected the expression patterns of lncRNAs and miRNAs to be important information for predicting interactions.

How can known information be used to effectively predict lncRNA-miRNA interactions? This problem is essentially a linkage prediction problem for the interactions network (Lü \& Zhou 2011). Previous studies have focused on improving the accuracy of prediction methods, without investigating the extent to which lncRNA-miRNA interactions can be predicted. In this study, we refer to the model proposed by Lü et al. (2015) and use a structural consistency index to measure the predictability of interactions. Given the adjacency matrix of a network, its first-order matrix perturbation can describe the inherent properties of the network structure and can reflect the link predictability without prior knowledge of the network organization. That is, the consistency of structural features before and after the removal of a random component of the links reflects the predictability of a network. A model based on the structural perturbation 
105 method was developed to predict lncRNA-miRNA interactions. We refer to this model as

106

107

108

109

110

111

112

113

114

115

116

117

118

119

120

121

122

123

124

125

126

127

128

129

130

131

132

133

134

135

136

137

138

SPMLMI (i.e., Structural Perturbation Method for predicting LncRNA-MiRNA Interactions).

This study makes several key contributions. First, we constructed an lncRNA-miRNA bilayer network composed of three kinds of information (lncRNA similarity, miRNA similarity, and the validated lncRNA-miRNA interactions). Second, we used the structural consistency index to measure the link predictability of multiple related networks and found that the structural consistency of the bilayer network was greater than that of any of single network, supporting the use of the bilayer network for link prediction. Furthermore, we employed SPMLMI to predict potential lncRNA-miRNA interactions. Last, experimental results showed that the newly developed method achieves good prediction performance using different datasets and that its generalizability is better than those of many previous methods. Overall, SPMLMI is an effective method for predicting unknown lncRNA-miRNA interactions.

\section{Materials \& Methods}

\section{Data collection}

Our goal was to develop a method that is able to predict candidate interactions between lncRNAs and miRNAs. Three kinds of data were collected from various databases and a bilayer network of lncRNAs and miRNAs was constructed to represent the complex relationships. First, known lncRNA-miRNA interaction data were downloaded from the lncRNASNP database version 2.0 (http://bioinfo.life.hust.edu.cn/lncRNASNP) (Miao et al. 2018) and used to construct an lncRNA-miRNA network. Second, expression data for lncRNAs were downloaded from the NONCODE database (http://www.noncode.org/) (Fang et al. 2018) and used to calculate the expression similarity between lncRNAs and to construct the lncRNA similarity network. Additionally, miRNA expression data were downloaded from the miRmine database (http://guanlab.ccmb.med.umich.edu/mirmine) (Panwar et al. 2017) and used to construct the miRNA similarity network. Table 1 shows the data obtained from publicly available databases.

\section{Construction of an IncRNA-miRNA bilayer network}

An lncRNA-miRNA bilayer network containing two kinds of nodes (miRNAs and lncRNAs) and three types of relationships (miRNA-miRNA similarity relationship, lncRNA-lncRNA similarity relationship, as well as the known lncRNA-miRNA interaction relationship) was constructed.

To construct the lncRNA-miRNA interaction network, data were downloaded from the version 2.0 of the lncRNASNP database (Miao et al. 2018). This database contains known 
139 lncRNA-miRNA interactions with experimental validation collected from version 2.0 of the 140 starBase (Li et al. 2014). Then, we define matrix LMnet as the known IncRNA-miRNA

141 interaction network, where if lncRNA $i$ and miRNA $j$ are connected, the element LMnet $=1$;

142 otherwise, LMnet $=0$. A total of 39366 associations between $3150 \operatorname{lncRNAs}$ and 262 miRNAs

143 were retained after removing duplicates. Figure 1(b) presents a simple example of lncRNA-

144 miRNA interaction network construction.

145 The lncRNA similarity network and miRNA similarity network were constructed based on 146 expression profiles. First, expression profile data for miRNAs were downloaded from the 147 miRmine database (Panwar et al. 2017). The database contains data for 2588 miRNAs in 135 148 different human tissues and cell lines. After removing missing items and matching the miRNA 149 IDs in the lncRNA-miRNA interaction network ( LMnet), expression data for 262 miRNAs in 124 different human tissues and cell lines were retained. Second, lncRNA expression profiles were

151

152

153

154

155

156

157

158

159

160

161

162

163

164

165

166

167

168

169

170 downloaded from the NONCODE database (Fang et al. 2018). Expression profiles of 171814 transcripts in 16 different human tissues and 8 cell lines were obtained. After the removal of duplicates and matching lncRNA IDs in LMnet, 3150 lncRNA expression records for 24 different human tissues and cell lines were retained.

To describe the expression characteristics of ncRNAs in different tissues or cell lines in an unbiased fashion, the expression profiles were standardized before calculating pairwise similarity values. The Pearson correlation coefficient was used to measure the similarity of ncRNAs and the expression similarity matrix of $\operatorname{lncRNAs}$ and miRNAs was obtained. The similarity matrix represented the similarity network of these two types of ncRNAs, referred to as LSnet and MSnet, respectively, as shown in Figure 1 (a) and (c). For a pair of RNAs, a higher correlation indicates greater similarity in expression, in general.

Finally, the above three networks are integrated to build a bilayer network. Specifically, the lncRNA-miRNA bilayer network can be represented by a block adjacency matrix $A \in R^{N \times N}$,

$$
A=\left[\begin{array}{cc}
\text { LSnet } & \text { LMnet } \\
\text { LMnet }^{T} & \text { MSnet }
\end{array}\right],
$$

where $N=3412$ (the total number of nodes in the network, $3150 \operatorname{lncRNAs}$ and $262 \mathrm{miRNAs}$ ) and LMnet $^{T}$ is the transpose of LMnet .

Overall, the lncRNA-miRNA bilayer network was composed of the miRNA similarity network, lncRNA similarity network, and edges connecting the two networks. Figure 1 demonstrates the workflow for constructing the bilayer network and its matrix representation.

\section{Structural consistency and structural perturbation method}


171 Structural consistency was first proposed by Lü et al. (2015) and can be used to evaluate the 172 likelihood of links prediction in complex network. It is defined as the consistency of network 173 structural features before and after the elimination of partial associations at random. For the 174 rationale and the specific derivation, refer to the supplementary material

175 (Supplementary_File_S1). In this study, this method was applied to the IncRNA-miRNA bilayer 176 network $A$ and its sub-networks (LSnet and MSnet) and the structural consistency of each network 177 was evaluated separately.

178 Generally, the link prediction problem refers to the problem of estimating the probability of 179 the existence of unobserved links according to known topological information. The network 180 structure perturbation method involved in the structure consistency calculation process can be 181 used to predict missing links (Lü et al., 2015). After obtaining the lncRNA-miRNA bilayer 182 network (adjacency matrix, $A$ ) in Section 2.2, we derive the perturbation matrix $A^{\prime}$ by the 183 structural perturbation method. The prediction matrix $\hat{A}^{\prime}$ is then obtained by averaging $t$ 184 independent perturbation calculations. See the supplementary materials for more details 185 (Supplementary_File_S1). In this way, elements in the prediction matrix $\hat{A}^{\prime}$ can be interpreted as scores for each pair of nodes in the lncRNA-miRNA bilayer network. The scores in $\hat{A}^{\prime}$ determine the extent of all unobserved lncRNA-miRNA interactions, and we assume that a higher score corresponds to a greater likelihood of a potential interaction.

\section{Evaluation metrics}

To evaluate the performance of SPMLMI, $k$-fold cross-validation was performed based on verified lncRNA-miRNA interactions downloaded from the lncRNASNP database (Miao et al. 2018). In the cross-validation process, the original set was randomly divided into $k$ equally sized subsets. Among these $k$ subsets, one was used as the validation data for testing the model, and the others were used as the training data. Specifically, we randomly reset $1 / \mathrm{k}$ known interaction entries of the training dataset to unknown, and prioritize the candidate interaction entries in the prediction matrix by SPMLMI scores. The known interactions are considered as positive, and the rest as negative. True positive rate is the proportion of overlap between the top $1 / \mathrm{k}$ ranked candidate interactions with the known interactions. Then, the cross-validation was repeated $k$ times, taking each of the $k$ subsets as the validation data in turn. The cross-validation results are averaged to produce a single estimation.

The AUC (area under the receiver operating characteristic curve) was estimated to evaluate the performance of IncRNA-miRNA interaction prediction results. In particular, the TPR (true positive rate) and FPR (false positive rate) were calculated by varying the threshold and the ROC (receiver operating characteristic) curve and AUC value were obtained, where TPR and FPR represent the percentage of test samples above or below the given thresholds, respectively. 
207

208

209

210

\section{Results}

\section{Structural consistency of different networks}

213

214

215

216

217

218

219

220

221

222

223

224

225

226

227

228

229

230

231

232

233

234

235

236

237 performance. newly established or novel interactions).

\section{Performance evaluation and parameter optimization}

In addition, we applied SPMLMI to datasets analyzed by two additional methods, EPLMI (Huang et al. 2018) and SNFHGILMI (Fan et al. 2020), for an objective comparison of

We calculated the structural consistency of three datasets (i.e., the EPLMI dataset provided by Huang, et al. (2018), SNFHGILMI dataset provided by Fan, et al. (2020), and SPMLMI dataset obtained in this study). Each group of datasets was composed of an miRNA similarity network ( MSnet ), an lncRNA similarity network ( LSnet ), and a bilayer network ( Bilayer - net ). We randomly selected $10 \%$ of the total links from each network as the perturbation set (note that the bilayer network contains two kinds of links). As shown in Figure 2 (a), in three different datasets, the structural consistency values for the lncRNA-miRNA bilayer network ( Bilayer-net, yellow bar) were $0.2413 \pm 0.0015,0.3036 \pm 0.0011$, and $0.2168 \pm 0.0005$, respectively. The structural consistency of these three bilayer networks ( Bilayer-net ) were all greater than that of the similarity network (where the blue bar is MSnet and the orange bar is LSnet in Figure 2), indicating that considering more information can improve the structural consistency of the network. These results show that the construction of the bilayer network improves the inherent link predictability of the network and will be helpful for the prediction of "missing links" (i.e.,

First, to evaluate the performance of our proposed SPMLMI for the prediction of lncRNAmiRNA interactions, we adopted 2-fold, 5-fold and 10-fold cross-validation frameworks. To avoid the bias of sample division in cross-validation, we repeated each experiment 10 times and took the average value. Figure 2 (c) shows the corresponding ROC curve for each k-fold crossvalidation. The average areas under the curves were $0.8617 \pm 0.0013,0.9512 \pm 0.0034$, and $0.9517 \pm 0.0016$ for 2 -fold, 5 -fold, and 10-fold cross-validation, respectively. These results indicate that $k$-fold cross-validation increase improves the performance of SPMLMI. There was no significant difference (paired $t$-test, $p$-value $=0.1997$ ) between 5 -fold and 10-fold crossvalidation. Therefore, in subsequent experiments, we use 5-fold cross-validation to save time. 
238

239

240

241

242

243

244

245

246

247

248

249

250

251

252

253

254

255

256

257

258

259

260

261

262

263

264

265

266

267

268

269

270

271

272

273

Second, to optimize $t$ (i.e., the number of perturbations), we tuned the parameter from 1 to 16 . Figure 2 (b) shows boxplots of the variation of AUC values with increasing $t$. The AUC values increased as $t$ increased; however, for values of $t$ greater than or equal to 8 , AUC was relatively stable. For simplicity, we set the parameter $t$ to 8 for subsequent experiments.

\section{Comparison with previous methods}

Furthermore, we compared SPMLMI with the previously established EPLMI (Huang et al., 2018) based on the two-way diffusion algorithm and SNFHGILMI (Fan et al., 2020) based on the heterogeneous graph inference method. Initially, we evaluated the same dataset (i.e., the SPMLMI dataset obtained in this study, see Section 2 for details) by these three methods. Figure 3 (a) shows the ROC curves for the prediction performance of these three models. The average AUC for SPMLMI was 0.9512 \pm 0.0034 , which was higher than the AUC values of $0.7999 \pm$ 0.0014 for EPLMI and $0.5163 \pm 0.0013$ for SNFHGILMI, respectively. We also compared the methods using the original datasets. Using the EPLMI dataset provided by Huang, et al. (2018), the average AUC values for the three methods (SPMLMI, EPLMI, and SNFHGILMI) were $0.8767 \pm 0.0033,0.8417 \pm 0.0017$, and $0.5184 \pm 0.0023$, respectively (Figure 3 (b)). For the SNFHGILMI dataset provided by Fan et al. (2020), the average AUC values were $0.8653 \pm$ $0.0021,0.8570 \pm 0.0012$, and $0.9426 \pm 0.0035$, respectively (Figure 3 (c)).

These results prove that the newly established SPMLMI has good performance and generalizability across datasets.

\section{Case studies}

Two lncRNAs, SNHG14 (UBE3A-ATS/NONHSAT041137) and MALAT1 (NONHSAT022132), with important biological functions were selected to validate the prediction performance of SPMLMI. SNHG14, also known as UBE3A-ATS, overlaps with the entire $U B E 3 A$ gene and its paternal expression is thought to negatively regulate paternal $U B E 3 A$ expression in neurons, thereby determining tissue-specific imprinting of $U B E 3 A$ in the brain (Galiveti et al. 2014; Stanurova et al. 2016). MALA1 is located less than $70 \mathrm{~kb}$ from NCRNA00084 on chromosome 11q13.1 (Hutchinson et al. 2007) and is dysregulated in many diseases, such as alcohol-related diseases, endometrioid endometrial carcinoma, and non-small cell lung cancer (Ji et al. 2003; Kryger et al. 2012; Li et al. 2016). For each of this two lncRNAs in the interaction network, known interaction entries were randomly reset to unknown. We implemented SPMLMI using the reset data set and prioritized all candidate miRNAs according to their scores in the prediction matrix.

As shown in Table 2, among the top 10\% (26) predicted SNHG14-related miRNAs, 18 lncRNA-miRNA interactions were confirmed by experimental validation (containing 17 of the 
27421 known interactions we collected from the lncRNASNP2 database (Miao et al. 2018)). It 275 should be noted that the miRNA hsa-miR-126-3p (marked with an asterisk in Table 2), ranked 276 16th, was not collected from the lncRNASNP2 database (Miao et al. 2018) but was reported by 277 an experimental study of transcriptome-wide Ago2:RNA interactions in human brain tissues 278 using HITS-CLIP (i.e., CLIP-seq) (Boudreau et al. 2014). This information was retrieved from 279 DIANA-LncBase v3 (Karagkouni et al. 2020). Several studies have shown that miR-126 directly 280 targets the 3'-UTR of insulin receptor substrate-1 (IRS-1) (Ryu et al. 2011; Zhang et al. 2008; 281 Zhou et al. 2013).Over-expression of miR-126 leads to downregulation of IRS-1 expression, and

282 283 284 285 286 287 288 289

290

291

292

293

294

295

296

297

298

299

300

301

302

303

304

305

306

307

308 a consequent impairment in insulin signaling (Ryu et al. 2011). Moreover, the research results of Zhou et al. (2013) showed over-expression of miR-126 down-regulated IRS-1, suppressed AKT and ERK1/2 activation, CRC cells proliferation, migration, invasion, and caused cell cycle arrest. Therefore, lncRNA SNHG14, as an interacting molecule of miR-126, may play a potential role in regulating cell metabolism and cell cycle.

Similarly, for MALAT1, we examined the candidate miRNAs ranked by prediction scores. The results showed that the top $10 \%$ of predicted MALAT1-related miRNAs were all verified in published studies (Supplementary_Table_S1). The reason is most likely that lncRNA MALAT1 has more known interaction information (containing 113 known interactions collected from the lncRNASNP2 database (Miao et al. 2018)). Therefore, we examined more predicted candidate miRNAs and found that a total of 106 were verifiable. Additionally, in a genome-wide analysis of miRNA-mRNA interactions in marrow stromal cells, the interaction between lncRNA MALAT1 and miRNA hsa-miR-487b-3p was confirmed (Balakrishnan et al. 2014); this candidate miRNA was ranked 91st (Supplementary_Table_S1) based on prediction scores. The experimental study by $\mathrm{Xi}$ et al. (2013) demonstrated that miR-487b directly targets gene Wnt5a. The protein Wnt5a is an important activator of the $\mathrm{Wnt} / \mathrm{Ca}^{2+}$ signaling pathway, which plays an important role in embryonic development, cell differentiation, inflammation and other physiological processes (De 2011). Because of sharing miRNA miR-487b with Wnt5a gene, lncRNA MALAT1 may also be involved in this complex signaling network.

The above results suggest that SPMLMI has good prediction ability and can be used to screen candidate target miRNAs for a given lncRNA.

\section{Discussion}

The identification of associations among biomolecules has important implications for our understanding of biological processes. In view of the complexity of intermolecular interaction networks, it is necessary to establish a prediction model that makes full use of prior information. Lü et al. (2015) studied the link predictability of complex networks and proposed the structural 
309 perturbation method for predicting "missing links" in networks. Zeng et al. (2018) applied this 310 algorithm to biological networks. They constructed a bilayer network and predicted unknown 311 miRNA-disease associations by the perturbation of specific diseases in the network, achieving 312 good results. We further extended the structural perturbation method; the key difference in our 313 study was the application of structural perturbation to the entire known IncRNA-miRNA 314 interaction network, without focusing on a specific ncRNA (lncRNA or miRNA).

315 The ceRNA hypothesis (Salmena et al. 2011) predicts that similarity in the expression profile 316 of a pair of lncRNAs is beneficial if they interact with the same miRNA. In our study, the 317 successful prediction of lncRNA-miRNA interactions based on ncRNA expression profile 318 similarity seems to support this hypothesis; however, the reality is more complex for a few 319 reasons. First, the ncRNA expression profile data used in this study were collected from different 320 human tissues and cell lines (see section 2.2). The similarity network reflects the generalized 321 expression pattern of a pair of ncRNAs, rather than their expression pattern in a specific cellular 322 environment. Second, an interaction between an IncRNA and miRNA may involve other 323 molecules. Because lncRNAs are abundant in vivo and have various interactions with other 324 molecules (Liu et al. 2017; Liu et al. 2015). Therefore, the indirect interactions of lncRNAs and miRNAs via other molecules, such as protein complexes, cannot be ignored.

Based on our results, we suggest that the network structure perturbation method has two main advantages over other approaches: (1) it can make full use of prior information in the network (2) and it captures network topology features well. However, the method has limitations. First, it requires prior knowledge of network nodes and links to achieve good performance. Second, our predicted candidate interaction pairs may contain indirect relationships involving other molecules (e.g., proteins, nucleic acids, etc.). The above summary of advantages and disadvantages are aspects of the method that should be noted, and also point out a direction we need to further research in the future. In addition, previous researchers have many excellent research work on miRNA-disease associations (Chen et al. 2019b) as well as lnRNA-disease associations (Chen \& Yan 2013). The studies of ncRNA-disease association by Chen et al. (2013; 2019b) inspired us to expand ncRNA-disease association studies by combining a priori information and reliable lncRNA-miRNA prediction information in our future research work.

Overall, the approach is valuable for large biomolecular networks and the generalized prediction of unknown links in the network or for analyses of specific molecules in a network.

\section{Conclusions}


343

344

345

346

347

348

349

350

351

352

353

354

355

356

357

358

359

\section{0}

361

362

363

364

365

366

367

368

369

370

371

372

373

374

375

We propose a framework named SPMLMI based on a bilayer network to predict interactions between lncRNAs and miRNAs. The bilayer network integrates similarity information for two types of ncRNA molecules and known interaction information. By using SPMLMI, we obtained a perturbation matrix and corresponded each matrix element to the correlation score between pairs of nodes in the network. In this way, missing links (i.e., unknown lncRNA-miRNA interactions) in the network are assigned correlation scores, where high scores indicate probable interactions. SPMLMI was feasible and effective for predicting lncRNA-miRNA interactions and represents a substantial improvement over SPMLMI.

Extensive regulatory functions of ncRNAs have been confirmed. Based on expression data, our method infers potential interactions between lncRNAs and miRNAs, providing a basis for further functional analyses. However, other regulatory interactions, such as $\operatorname{lncRNA}-\mathrm{mRNA}$ interactions, miRNA-mRNA interactions, and ncRNA-protein interactions, have not been considered and will be a focus of future research.

\section{Data availability}

The codes and datasets are available online at https://github.com/xumingmin/SPMLMI.git

\section{Acknowledgements}

\section{References}

Ala U, Karreth FA, Bosia C, Pagnani A, Taulli R, Leopold V, Tay Y, Provero P, Zecchina R, and Pandolfi PP. 2013. Integrated transcriptional and competitive endogenous RNA networks are cross-regulated in permissive molecular environments. Proc Natl Acad Sci U S A 110:7154-7159 DOI: 10.1073/pnas.1222509110.

Balakrishnan I, Yang X, Brown J, Ramakrishnan A, Torok-Storb B, Kabos P, Hesselberth JR, and Pillai MM. 2014. Genome-wide analysis of miRNA-mRNA interactions in marrow stromal cells. Stem Cells 32:662-673 DOI: 10.1002/stem.1531.

Béthune J, Artus-Revel CG, and Filipowicz W. 2012. Kinetic analysis reveals successive steps leading to miRNA-mediated silencing in mammalian cells. EMBO Rep 13:716-723 DOI: 10.1038/embor.2012.82.

Boudreau RL, Jiang P, Gilmore BL, Spengler RM, Tirabassi R, Nelson JA, Ross CA, Xing Y, and Davidson BL. 2014. Transcriptome-wide discovery of microRNA binding sites in human brain. Neuron 81:294-305 DOI: 10.1016/j.neuron.2013.10.062. 
376

377

378

379

380

381

382

383

384

385

386

387

388

389

390

391

392

393

394

395

396

397

398

399

400

401

402

403

404

405

406

407

408

409

410

411

412

413

414

415

416

Braun JE, Huntzinger E, and Izaurralde E. 2013. The Role of GW182 Proteins in miRNA-Mediated Gene Silencing. In: Chan EKL, and Fritzler MJ, eds. Ten Years of Progress in GW/P Body Research. New York, NY: Springer New York, 147-163.

Cech TR, and Steitz JA. 2014. The noncoding RNA revolution-trashing old rules to forge new ones. Cell 157:77-94 DOI: 10.1016/j.cell.2014.03.008.

Chen X, and Yan GY. 2013. Novel human lncRNA-disease association inference based on lncRNA expression profiles. Bioinformatics 29:2617-2624 DOI: 10.1093/bioinformatics/btt426.

Chen X, Sun YZ, Guan NN, Qu J, Huang ZA, Zhu ZX, and Li JQ. 2019a. Computational models for lncRNA function prediction and functional similarity calculation. Brief Funct Genomics 18:58-82 DOI: 10.1093/bfgp/ely031.

Chen X, Xie D, Zhao Q, and You ZH. 2019b. MicroRNAs and complex diseases: from experimental results to computational models. Brief Bioinform 20:515-539 DOI: 10.1093/bib/bbx130.

Chen X, Yan CC, Zhang X, and You ZH. 2017. Long non-coding RNAs and complex diseases: from experimental results to computational models. Brief Bioinform 18:558-576 DOI: 10.1093/bib/bbw060.

Clauset A, Moore C, and Newman ME. 2008. Hierarchical structure and the prediction of missing links in networks. Nature 453:98-101 DOI: 10.1038/nature06830.

De A. 2011. Wnt/Ca2+ signaling pathway: a brief overview. Acta Biochim Biophys Sin (Shanghai) 43:745-756 DOI: 10.1093/abbs/gmr079.

Fan YX, Cui J, and Zhu QQ. 2020. Heterogeneous graph inference based on similarity network fusion for predicting lncRNA-miRNA interaction. Rsc Advances 10:11634-11642 DOI: $10.1039 / \mathrm{c} 9 \mathrm{ra} 11043 \mathrm{~g}$.

Fang S, Zhang L, Guo J, Niu Y, Wu Y, Li H, Zhao L, Li X, Teng X, Sun X, Sun L, Zhang MQ, Chen R, and Zhao Y. 2018. NONCODEV5: a comprehensive annotation database for long non-coding RNAs. Nucleic Acids Res 46:D308-D314 DOI: 10.1093/nar/gkx1107.

Galiveti CR, Raabe CA, Konthur Z, and Rozhdestvensky TS. 2014. Differential regulation of non-protein coding RNAs from Prader-Willi Syndrome locus. Sci Rep 4:6445 DOI: 10.1038/srep06445.

Guil S, and Esteller M. 2015. RNA-RNA interactions in gene regulation: the coding and noncoding players. Trends Biochem Sci 40:248-256 DOI: 10.1016/j.tibs.2015.03.001.

Huang YA, Chan KCC, and You ZH. 2018. Constructing prediction models from expression profiles for large scale lncRNA-miRNA interaction profiling. Bioinformatics 34:812-819 DOI: 10.1093/bioinformatics/btx672.

Huntzinger E, and Izaurralde E. 2011. Gene silencing by microRNAs: contributions of translational repression and mRNA decay. Nat Rev Genet 12:99-110 DOI: 10.1038/nrg2936.

Hutchinson JN, Ensminger AW, Clemson CM, Lynch CR, Lawrence JB, and Chess A. 2007. A screen for nuclear transcripts identifies two linked noncoding RNAs associated with SC35 splicing domains. BMC Genomics 8:39 DOI: 10.1186/1471-2164-8-39.

Ji P, Diederichs S, Wang W, Boing S, Metzger R, Schneider PM, Tidow N, Brandt B, Buerger H, Bulk E, Thomas M, Berdel WE, Serve H, and Muller-Tidow C. 2003. MALAT-1, a novel noncoding RNA, and thymosin beta4 predict metastasis and survival in early-stage non-small cell lung cancer. Oncogene 22:8031-8041 DOI: 10.1038/sj.onc.1206928 
417 Karagkouni D, Paraskevopoulou MD, Tastsoglou S, Skoufos G, Karavangeli A, Pierros V,

418 Zacharopoulou E, and Hatzigeorgiou AG. 2020. DIANA-LncBase v3: indexing experimentally

419

420

421

422

423

424

425

426

427

428

429

430

431

432

433

434

435

436

437

438

439

440

441

442

443

444

445

446

447

448

449

450

451

452

453

454

455

456 supported miRNA targets on non-coding transcripts. Nucleic Acids Res 48:D101-D110 DOI: 10.1093/nar/gkz1036.

Krol J, Loedige I, and Filipowicz W. 2010. The widespread regulation of microRNA biogenesis, function and decay. Nat Rev Genet 11:597-610 DOI: 10.1038/nrg2843.

Kryger R, Fan L, Wilce PA, and Jaquet V. 2012. MALAT-1, a non protein-coding RNA is upregulated in the cerebellum, hippocampus and brain stem of human alcoholics. Alcohol 46:629-634 DOI: 10.1016/j.alcohol.2012.04.002.

Lai D, and Meyer IM. 2016. A comprehensive comparison of general RNA-RNA interaction prediction methods. Nucleic Acids Res 44:e61 DOI: 10.1093/nar/gkv1477.

Lee RC, Feinbaum RL, and Ambros V. 1993. The C. elegans heterochronic gene lin-4 encodes small RNAs with antisense complementarity to lin-14. Cell 75:843-854 DOI: 10.1016/00928674(93)90529-y.

Li J, Ma W, Zeng P, Wang J, Geng B, Yang J, and Cui Q. 2015. LncTar: a tool for predicting the RNA targets of long noncoding RNAs. Brief Bioinform 16:806-812 DOI: 10.1093/bib/bbu048.

Li JH, Liu S, Zhou H, Qu LH, and Yang JH. 2014. starBase v2.0: decoding miRNA-ceRNA, miRNAncRNA and protein-RNA interaction networks from large-scale CLIP-Seq data. Nucleic Acids Res 42:D92-97 DOI: 10.1093/nar/gkt1248.

Li Q, Zhang C, Chen R, Xiong H, Qiu F, Liu S, Zhang M, Wang F, Wang Y, Zhou X, Xiao G, Wang X, and Jiang Q. 2016. Disrupting MALAT1/miR-200c sponge decreases invasion and migration in endometrioid endometrial carcinoma. Cancer Lett 383:28-40 DOI: 10.1016/j.canlet.2016.09.019.

Liu CJ, Gao C, Ma Z, Cong R, Zhang Q, and Guo AY. 2017. IncRInter: A database of experimentally validated long non-coding RNA interaction. J Genet Genomics 44:265-268 DOI: 10.1016/j.jgg.2017.01.004.

Liu N, Dai Q, Zheng G, He C, Parisien M, and Pan T. 2015. N(6)-methyladenosine-dependent RNA structural switches regulate RNA-protein interactions. Nature 518:560-564 DOI: 10.1038/nature14234.

Lü L, Pan L, Zhou T, Zhang YC, and Stanley HE. 2015. Toward link predictability of complex networks. Proc Natl Acad Sci US A 112:2325-2330 DOI: 10.1073/pnas.1424644112.

Lü LY, and Zhou T. 2011. Link prediction in complex networks: A survey. Physica a-Statistical Mechanics and Its Applications 390:1150-1170 DOI: 10.1016/j.physa.2010.11.027.

Lv D, Xu K, Jin X, Li J, Shi Y, Zhang M, Jin X, Li Y, Xu J, and Li X. 2020. LncSpA: LncRNA Spatial Atlas of Expression across Normal and Cancer Tissues. Cancer Res 80:2067-2071 DOI: 10.1158/0008-5472.CAN-19-2687.

Miao YR, Liu W, Zhang Q, and Guo AY. 2018. lncRNASNP2: an updated database of functional SNPs and mutations in human and mouse lncRNAs. Nucleic Acids Res 46:D276-D280 DOI: 10.1093/nar/gkx1004.

Morris KV, and Mattick JS. 2014. The rise of regulatory RNA. Nat Rev Genet 15:423-437 DOI: $10.1038 / \operatorname{nrg} 3722$. 
457 Panwar B, Omenn GS, and Guan Y. 2017. miRmine: a database of human miRNA expression profiles.

458

459

460

461

462

463

464

465

466

467

468

469

470

471

472

473

474

475

476

477

478

479

480

481

482

483

484

485

486

487

488

489

490

491

492

493

494

495

496

497 Bioinformatics 33:1554-1560 DOI: 10.1093/bioinformatics/btx019.

Quinn JJ, and Chang HY. 2016. Unique features of long non-coding RNA biogenesis and function. Nat Rev Genet 17:47-62 DOI: 10.1038/nrg.2015.10.

Rinn JL, and Chang HY. 2012. Genome regulation by long noncoding RNAs. Annu Rev Biochem 81:145166 DOI: 10.1146/annurev-biochem-051410-092902.

Ryu HS, Park SY, Ma D, Zhang J, and Lee W. 2011. The induction of microRNA targeting IRS-1 is involved in the development of insulin resistance under conditions of mitochondrial dysfunction in hepatocytes. PLOS ONE 6:e17343 DOI: 10.1371/journal.pone.0017343.

Sabin LR, Delas MJ, and Hannon GJ. 2013. Dogma derailed: the many influences of RNA on the genome. Mol Cell 49:783-794 DOI: 10.1016/j.molcel.2013.02.010.

Salmena L, Poliseno L, Tay Y, Kats L, and Pandolfi PP. 2011. A ceRNA hypothesis: the Rosetta Stone of a hidden RNA language? Cell 146:353-358 DOI: 10.1016/j.cell.2011.07.014.

Stanurova J, Neureiter A, Hiber M, de Oliveira Kessler H, Stolp K, Goetzke R, Klein D, Bankfalvi A, Klump H, and Steenpass L. 2016. Angelman syndrome-derived neurons display late onset of paternal UBE3A silencing. Sci Rep 6:30792 DOI: 10.1038/srep30792.

Thomson DW, and Dinger ME. 2016. Endogenous microRNA sponges: evidence and controversy. Nat Rev Genet 17:272-283 DOI: 10.1038/nrg.2016.20.

Voinnet O. 2009. Origin, biogenesis, and activity of plant microRNAs. Cell 136:669-687 DOI: 10.1016/j.cell.2009.01.046.

Wightman B, Ha I, and Ruvkun G. 1993. Posttranscriptional regulation of the heterochronic gene lin-14 by lin-4 mediates temporal pattern formation in C. elegans. Cell 75:855-862 DOI: 10.1016/00928674(93)90530-4.

Xi S, Xu H, Shan J, Tao Y, Hong JA, Inchauste S, Zhang M, Kunst TF, Mercedes L, and Schrump DS. 2013. Cigarette smoke mediates epigenetic repression of miR-487b during pulmonary carcinogenesis. J Clin Invest 123:1241-1261 DOI: 10.1172/JCI61271.

Xu J, Feng L, Han Z, Li Y, Wu A, Shao T, Ding N, Li L, Deng W, Di X, Wang J, Zhang L, Li X, Zhang K, and Cheng S. 2016. Extensive ceRNA-ceRNA interaction networks mediated by miRNAs regulate development in multiple rhesus tissues. Nucleic Acids Res 44:9438-9451 DOI: 10.1093/nar/gkw587.

Xu J, Li Y, Lu J, Pan T, Ding N, Wang Z, Shao T, Zhang J, Wang L, and Li X. 2015. The mRNA related ceRNA-ceRNA landscape and significance across 20 major cancer types. Nucleic Acids Res 43:8169-8182 DOI: 10.1093/nar/gkv853.

Yoon JH, Abdelmohsen K, and Gorospe M. 2014. Functional interactions among microRNAs and long noncoding RNAs. Semin Cell Dev Biol 34:9-14 DOI: 10.1016/j.semcdb.2014.05.015.

Zeng X, Liu L, Lu L, and Zou Q. 2018. Prediction of potential disease-associated microRNAs using structural perturbation method. Bioinformatics 34:2425-2432 DOI:

10.1093/bioinformatics/bty112.

Zhang J, Du YY, Lin YF, Chen YT, Yang L, Wang HJ, and Ma D. 2008. The cell growth suppressor, mir-126, targets IRS-1. Biochem Biophys Res Commun 377:136-140 DOI: 10.1016/j.bbrc.2008.09.089. 
498 Zhou Y, Feng X, Liu YL, Ye SC, Wang H, Tan WK, Tian T, Qiu YM, and Luo HS. 2013. Down499 regulation of miR-126 is associated with colorectal cancer cells proliferation, migration and 500 invasion by targeting IRS-1 via the AKT and ERK1/2 signaling pathways. PLOS ONE 8:e81203 $501 \quad$ DOI: 10.1371 journal.pone.0081203. 


\section{Table $\mathbf{1}$ (on next page)}

Summary of data used in this study 
1

\begin{tabular}{lll}
\hline Database & Data types & Number of links/nodes \\
\hline \multirow{2}{*}{ lncRNASNP } & lncRNA-miRNA interactions & 39366 \\
& (validated) & (interactions/edges/links) \\
NONCODE & lncRNA expression profiles & 3150 \\
& & (lncRNA nodes) \\
miRmine & miRNA expression profiles & 262 \\
& & (miRNA nodes) \\
\hline
\end{tabular}

2 


\section{Table 2 (on next page)}

Prediction results for the top 10\% SNHG14-related miRNAs ranked by prediction scores. 


\begin{tabular}{|c|c|c|c|c|c|}
\hline Rank & MiRNAs & $\begin{array}{l}\text { Reference } \\
\text { (PMID) }\end{array}$ & Rank & MiRNAs & $\begin{array}{l}\text { Reference } \\
\text { (PMID) }\end{array}$ \\
\hline 1 & hsa-miR-425-5p & 23313552 & 14 & hsa-miR-32-5p & $\begin{array}{l}24668909 \\
28030800 \\
22291592\end{array}$ \\
\hline 2 & hsa-miR-181b-5p & $\begin{array}{l}23313552 ; \\
28030800 ; \\
22012620\end{array}$ & 15 & hsa-miR-92a-3p & $\begin{array}{l}24668909 \\
28030800 \\
22291592\end{array}$ \\
\hline 3 & hsa-miR-340-5p & $\begin{array}{l}24668909 \\
23313552\end{array}$ & 16 & hsa-miR-126-3p * & 24389009 \\
\hline 4 & hsa-miR-25-3p & $\begin{array}{l}24668909 \\
28030800\end{array}$ & 17 & hsa-miR-181c-5p & $\begin{array}{l}23313552 \\
28030800 ; \\
22012620\end{array}$ \\
\hline 5 & hsa-miR-363-3p & $\begin{array}{l}24668909 \\
28030800 \\
22291592\end{array}$ & 18 & hsa-miR-382-5p & 23313552 \\
\hline 6 & hsa-miR-543 & $\begin{array}{l}23313552 \\
28030800\end{array}$ & 19 & hsa-miR-551a & Unconfirmed \\
\hline 7 & hsa-miR-433-3p & 22012620 & 20 & hsa-miR-200c-3p & Unconfirmed \\
\hline 8 & hsa-miR-136-5p & 22012620 & 21 & hsa-miR-187-3p & Unconfirmed \\
\hline 9 & hsa-miR-181a-5p & $\begin{array}{l}23313552 \\
28030800 \\
22012620\end{array}$ & 22 & hsa-miR-378b & Unconfirmed \\
\hline 10 & hsa-miR-181d-5p & $\begin{array}{l}23313552 \\
28030800 \\
22012620\end{array}$ & 23 & hsa-miR-100-5p & Unconfirmed \\
\hline 11 & hsa-miR-376c-3p & $\begin{array}{l}24668909 \\
22100165\end{array}$ & 24 & hsa-miR-758-3p & Unconfirmed \\
\hline 12 & hsa-miR-206 & $\begin{array}{l}24906430 \\
22012620\end{array}$ & 25 & hsa-miR-10b-5p & Unconfirmed \\
\hline 13 & hsa-miR-92b-3p & $\begin{array}{l}24668909 \\
28030800 \\
22291592\end{array}$ & 26 & hsa-miR-300 & Unconfirmed \\
\hline
\end{tabular}




\section{Figure 1}

Flowchart of SPMLMI.

(a) The IncRNA expression profiles were downloaded from the NONCODE database and used to calculate the IncRNA similarity network (LSnet). (b) Known IncRNA-miRNA interactions were downloaded from the IncRNASNP database and used to construct the IncRNA-miRNA interaction network (LMnet). (c) The miRNA expression profiles were downloaded from the miRmine database and used to construct the miRNA similarity network (MSnet). (d) Three networks were integrated to construct the IncRNA-miRNA bilayer network. (e) The perturbed network was obtained by using SPMLMI. 


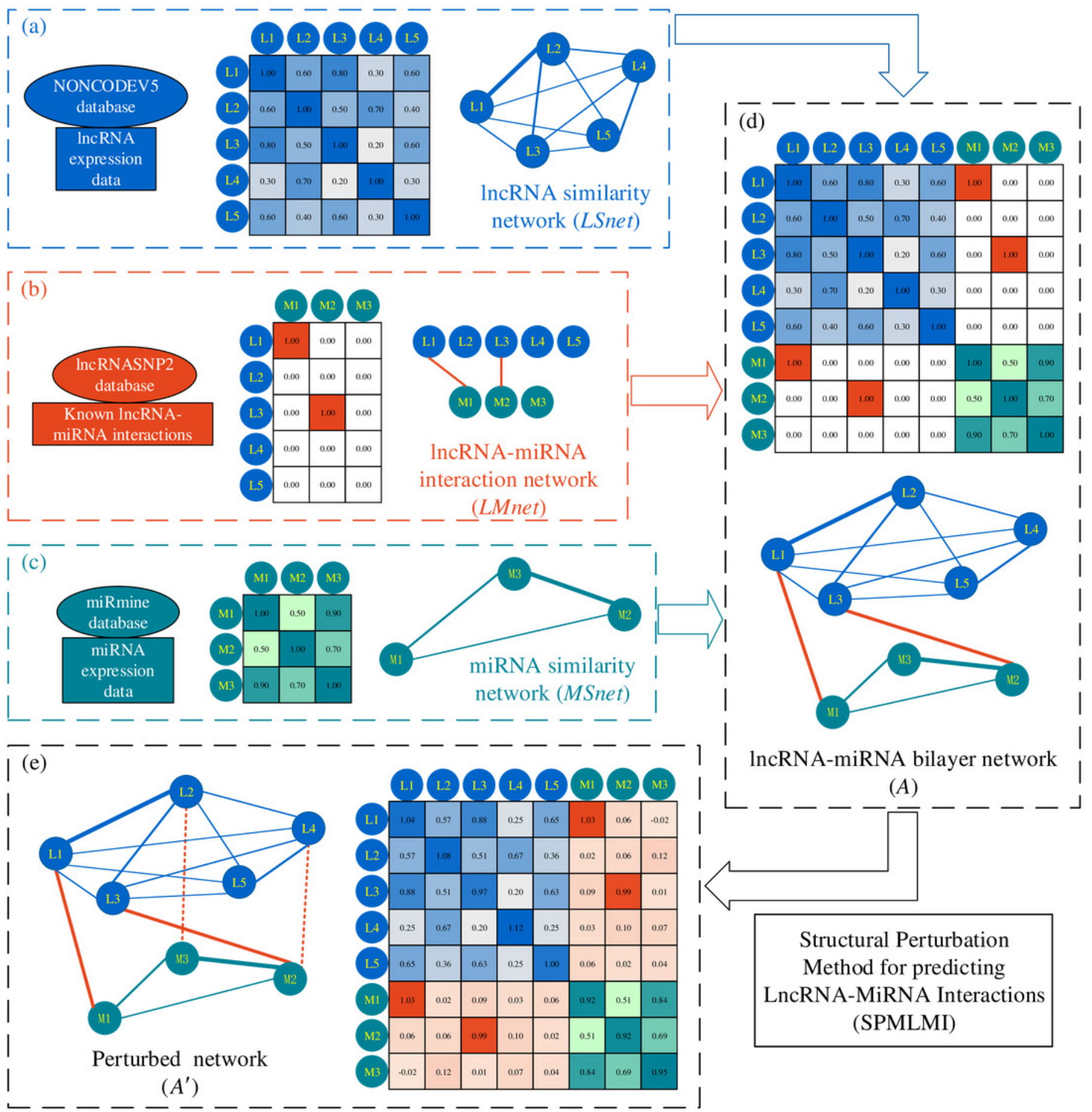


Figure 2

Experimental results on structural consistency, parameter $t$ optimization and performance evaluation.

(a) Structural consistency of related networks. (b) Effect of the parameter $t$ on model performance. (c) Performance of SPMLMI under different $k$-fold cross-validation frameworks.
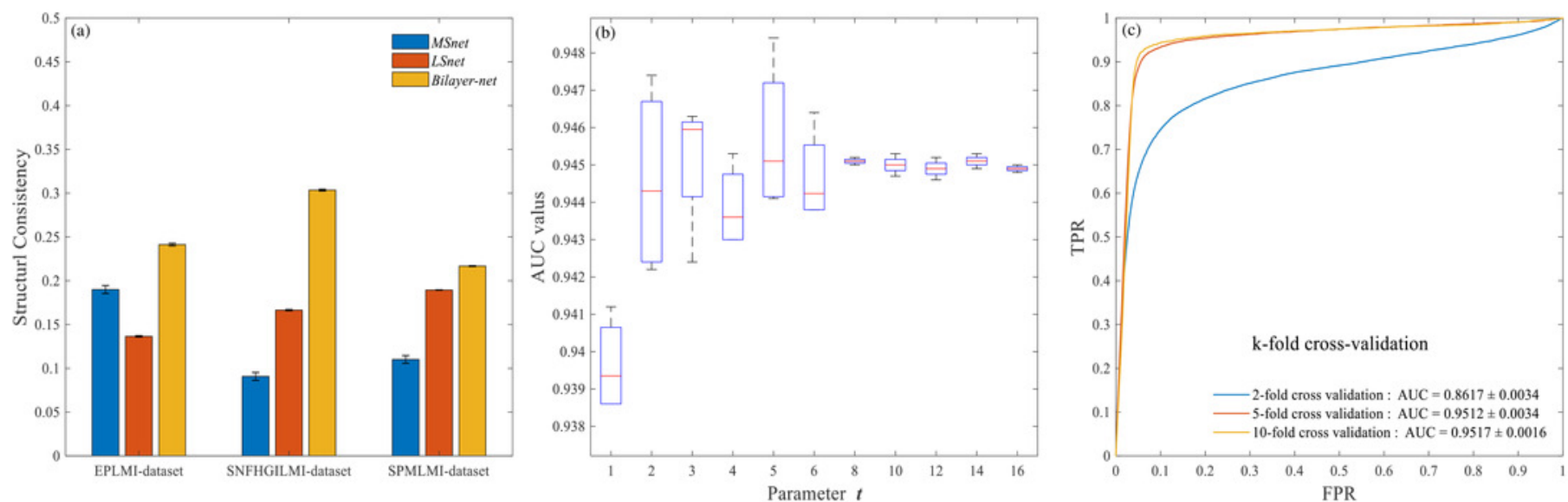
Figure 3

Comparison with previous methods.

(a) Experimental results for the three methods on the SPMLMI dataset. (b) Experimental results for the three methods on the EPLMI dataset. (c) Experimental results for three methods on the SNFHGILMI dataset.
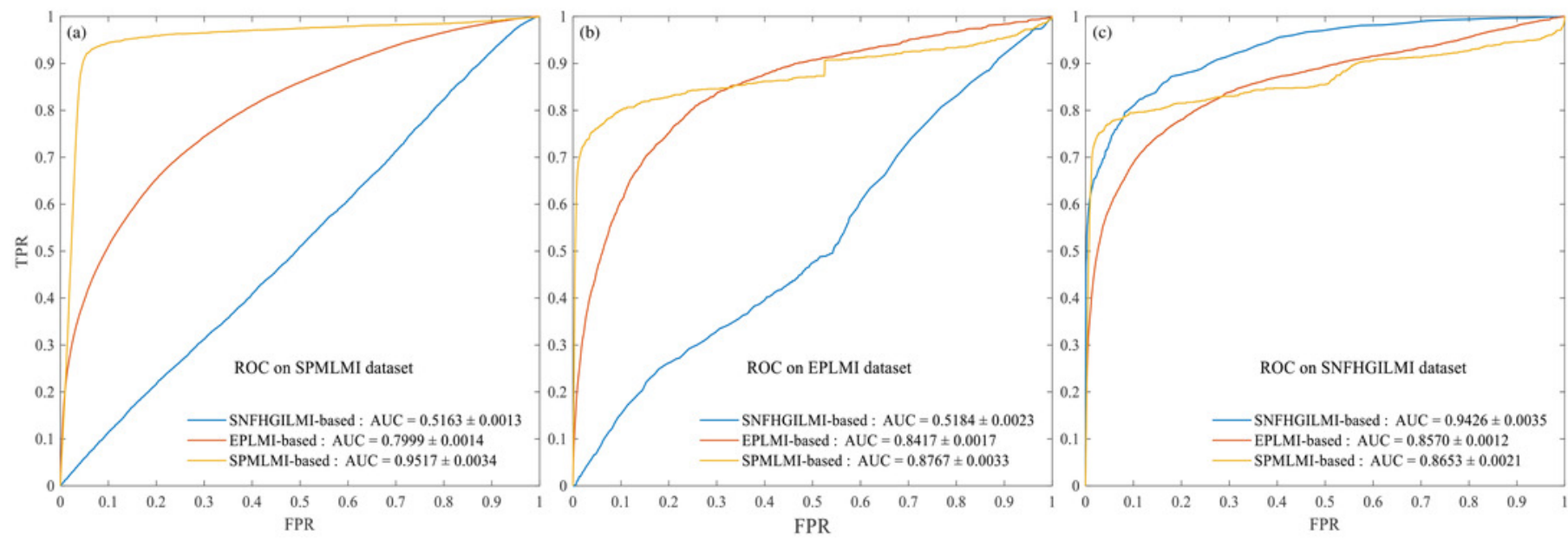